Espinosa-Herrera, G. (2017). Comportamientos diferenciados por género en el consumo de sustancias psicoactivas entre universitarios. Revista de Antropología y Sociología: VIRAJES, 19 (2), 171-184. DOI: 10.17151/rasv.2017.19.2.9

\title{
COMPORTAMIENTOS DIFERENCIADOS POR GÉNERO EN EL CONSUMO DE SUSTANCIAS PSICOACTIVAS ENTRE UNIVERSITARIOS*
}

\section{GRETEL ESPINOSA-HERRERA**}

Recibido: 10 de abril 2017

Aprobado: 10 de julio de 2017

\section{Artículo de Investigación}

\footnotetext{
* El presente artículo de investigación se deriva del trabajo sobre consumo de sustancias psicoactivas en universitarios que se desarrolló para los estudios del Doctorado en Ciencias Sociales, Niñez y Juventud (Universidad de Manizales-CINDE). Nombre del proyecto: “Estructuración de las prácticas de consumo de sustancias psicoactivas en estudiantes universitarios". Proyecto adscrito a la Vicerrectoría de Investigaciones de la Universidad de Caldas. Código 0701015.

** Doctora en Ciencias Sociales, Niñez y Juventud de la Universidad de Manizales-CINDE. Profesora Auxiliar del Departamento de Antropología y Sociología de la Universidad de Caldas. Manizales, Caldas. E-mail: gretel.espinosa@ucaldas.edu.co. (1) ORCID 0000-0003-1589-1304. Google Scholar
} 


\title{
Resumen
}

Objetivo. Exponer una reflexión acerca de los comportamientos diferenciados por género en el consumo de sustancias psicoactivas entre universitarios. Lo anterior, se sustenta a partir del caso de una universidad regional colombiana ${ }^{11}$. Metodología. Se aplica una metodología mixta, que utiliza un cuestionario de la CICAD/OEA, así como entrevistas con miembros de la misma institución. Resultados y conclusiones. Se argumenta que, si bien existen cambios en el consumo entre los jóvenes y en la percepción del consumo de sustancias psicoactivas por género, el rechazo social hacia las mujeres consumidoras sigue siendo un elemento diferenciador de la práctica.

Palabras clave: joven, género, universidad, sustancia alucinógena, consumo.

\section{BEHAVIOR DIFFERENCIATED BY GENDER IN THE CONSUMPTION OF PSYCHOACTIVE SUBSTANCES AMONG UNIVERISTY STUDENTS}

\begin{abstract}
Objective: To present a reflection on the differentiated behaviors by gender in the consumption of psychoactive substances among university students. The above is based on the case of a Colombian regional University. Methodology: A mixed methodology is applied using a questionnaire from CICAD/OEA as well as interviews with members of the same educational institution. Results and conclusions: It is argued that, although there are changes in consumption among young people and in the perception of the consumption of psychoactive substances by gender, the social rejection towards women consumers continues being a differentiating element of the practice.
\end{abstract}

Key words: Young person, gender, university, hallucinogenic substances, consumption (Unesco Social Sciences Thesaurus).

\footnotetext{
${ }^{1}$ La universidad regional que sirve de referente para este artículo es una universidad pública de carácter nacional que alberga a estudiantes de todos los rincones de Colombia y del exterior. En este sentido las universidades regionales buscan impactar en el desarrollo de sus regiones a partir de la cobertura en todas las áreas del conocimiento con enfoques acordes con la realidad de sus zonas de influencia.
} 


\section{Introducción}

1 consumo de drogas no es un hecho exclusivo y característico 4 de la contemporaneidad; su comprensión y explicación han variado en el tiempo y en el espacio; aunque es a partir del siglo XX que aparece el consumo de este tipo de sustancias como un comportamiento disfuncional y transgresor, regulado y explicado principalmente a través de los modelos médico y jurídico. Hoy en día, el consumo de sustancias psicoactivas es una conducta socialmente rechazada y sancionada ${ }^{1}$; sin embargo la percepción social sobre el consumo, así como los comportamientos asociados a este, varían de acuerdo con diferentes elementos que se relacionan con el género entre otros diferenciadores sociales.

$\mathrm{Al}$ respecto, algunos análisis sobre la variable género se acercan a la temática por medio del modelo médico y de disciplinas como la psicología para identificar una serie de factores de riesgo y protección frente al consumo de sustancias psicoactivas; los que estando presentes, elevan la probabilidad de que una persona desarrolle un comportamiento determinado (Pandina, 2002). En este orden de ideas, Laespada, Iraurgi y Arostege (2004) identifican tres grupos de factores de protección y de riesgo: factores personales, relacionales y sociales; y en cada uno de ellos el género aparece como una variable que transversaliza el asunto (Castellanos y Espinosa, 2013); sin embargo la ausencia de la perspectiva de género en el análisis hace que el nivel de conocimiento actual sobre algunos aspectos relacionados con el tema sea muy limitado, en especial en un momento donde coexisten los modelos tradicional y contemporáneo de los papeles femenino y masculino.

Frente a esta situación de coexistencia no hay que olvidar que las percepciones sobre los consumos de sustancias psicoactivas en función del género se relacionan con la edad de los sujetos. Las personas adultas, en su mayoría educadas en un modelo tradicional, perciben el consumo de mujeres como un comportamiento reprobable y objeto de rechazo social; percepción diferente entre los jóvenes, quienes ven como normal el hecho de que sus compañeras compartan los mismos comportamientos y hábitos (Espinosa-Herrera et al., 2016; Espinosa, Montoya y Beltrán, 2015).

\footnotetext{
${ }^{1}$ En el caso de Colombia la norma genera vacíos interpretativos, en tanto es contradictorio que el consumo de este tipo de sustancias sea sancionado al mismo tiempo que existe una ley que establece la legalidad para el porte y consumo de la dosis personal. Lo anterior unido a que, en mayo de 2016, se aprobó en el Congreso la ley que reglamenta el uso medicinal y terapéutico de la marihuana en todo el país. Con esta decisión Colombia se convirtió en el cuarto país de América Latina en tener legislación sobre el uso de la marihuana con fines terapéuticos y paliativos.
} 
Teniendo en cuenta lo anterior los contextos universitarios compuestos en su mayoría por población joven- son un escenario en el que, además de los procesos de formación académica y profesional que demanda el paso por la universidad, los jóvenes desafían otros procesos de construcción personal que conllevan a un sinnúmero de aprendizajes, prácticas y experiencias entre los que se encuentra el consumo de sustancias psicoactivas (Parra, 1996).

Así, este artículo presenta resultados de la investigación doctoral titulada "Estructuración de las prácticas de consumo de sustancias psicoactivas en un grupo de universitarios"; exponiendo una reflexión acerca del consumo de este tipo de sustancias, centrando la atención en los comportamientos diferenciados por género. Se argumenta que, si bien existen cambios en el consumo entre los jóvenes y en la percepción del consumo de sustancias psicoactivas por género, el rechazo social hacia las mujeres consumidoras sigue siendo un elemento diferenciador de la práctica.

\section{Aproximación teórica}

\section{Las sustancias psicoactivas}

A través de la historia han sido diversas las conceptualizaciones que explican lo que significan las drogas; sin embargo este trabajo equipara el término drogas, estigmatizado como problema social y médico, con el de sustancia psicoactiva: un término menos cargado de significado negativo y que se sitúa en los análisis de las perspectivas sociales. De esta manera se plantea que las sustancias psicoactivas son:

sustancias químicas que se incorporan al organismo humano, con capacidad para modificar varias funciones de éste (percepción, conducta, motricidad, etc.), pero cuyos efectos, consecuencias y funciones están condicionados, sobre todo, por las definiciones sociales, económicas y culturales que generan los grupos sociales que las utilizan. (Romani, 1999, p. 53)

Asimismo, "estas sustancias, mediante sus usos crean redes de intercomunicación y sociológicamente hablando, se constituyen en un dispositivo de socialización y de adaptación, según contextos y circunstancias, históricas y culturalmente establecidas@ (Ronderos, 1995, p. 3).

Dos aspectos importantes se destacan en estas enunciaciones: el primero se asocia con los efectos que una sustancia produce en el cuerpo 
humano y el otro se relaciona con los aspectos socioculturales que rodean el consumo de drogas, así como con los significados que las personas o grupos sociales construyen frente al consumo de este tipo de sustancias y a las dinámicas que se tejen en él.

Con base en ello hablar del consumo de drogas involucra acercarnos a la comprensión de un fenómeno social que implica pensar en una realidad particularizada de acuerdo con los diferentes contextos socioculturales, el momento del ciclo vital de los sujetos, sus condiciones socioeconómicas y su situación de género.

\section{El género como diferenciador de las prácticas de consumo de sustancias psicoactivas}

El género es un concepto construido socialmente que agrupa todos los aspectos psicológicos, sociales y culturales de lo femenino y lo masculino. El modelo tradicional de los roles que vinculaba a las mujeres con el espacio doméstico y a los hombres con lo público, condiciona socialmente los comportamientos y percepciones sobre las personas.

Nacer hombre o mujer implica vivir en contextos culturales con posiciones y recursos desiguales, así como con distintos valores, creencias y actitudes. La división de género imperante en cada sociedad asigna a hombres y mujeres roles o papeles sociales diferentes y determina un conjunto de pautas de comportamiento obligadas, permitidas o prohibidas, a las que deben ajustarse cada uno de estos grupos. Estas pautas de comportamiento son transmitidas y reproducidas a través de distintos espacios de socialización, fundamentalmente la familia, la escuela y los medios de comunicación social. Aunque la división de los roles femenino y masculino varía entre sociedades y en distintos momentos históricos, existe un modelo hegemónico (tradicional) de división de género del trabajo. (Sánchez, 2008, p. 117)

Esta división entre lo masculino y lo femenino implica la tendencia a que los hombres disfruten de mayor aceptación y reconocimiento social en diferentes temas de lo cotidiano entre ellos el consumo de sustancias psicoactivas, mientras que las mujeres reciben el señalamiento social y la estigmatización por "querer igualar sus comportamientos a los de los hombres".

En este sentido el género es, entonces, uno de los elementos que ha sido pensado como factor de riesgo y protección para el consumo de sustancias psicoactivas en relación con los significados construidos para hombres y 
mujeres, así como la valoración social desigual frente al consumo, en cada uno de los géneros.

Cabe señalar que en investigaciones previas la tendencia estaba marcada por un mayor consumo de sustancias psicoactivas, tanto legales como ilegales, por parte de los hombres; sin embargo, en años recientes esta realidad ha venido cambiando. Algunos estudios (Martínez, 2006; Fernández, 2010; Navarro, 2007) evidencian que las prácticas de consumo, respecto al género, han ido acortando paulatinamente esas marcadas diferencias al mostrar un acercamiento en las prevalencias de consumo. Pero no es posible afirmar que ambos comportamientos se igualen, sino que sugieren diferencias interesantes: (i) los hombres presentan porcentajes de consumo superiores a las mujeres. Así, la proporción de jóvenes consumidores que aumenta con la edad es mayor en los hombres jóvenes; (ii) la edad media de inicio en el consumo es ligeramente menor en los hombres; (iii) compartiendo las mismas condiciones familiares, los jóvenes hombres tienen más probabilidad de consumir drogas que sus hermanas; (iv) las mujeres no solo beben menos que los hombres, sino que también se diferencian en el tipo de bebida y en las circunstancias en las que beben; (v) el grado de consumo de drogas ilícitas recae fundamentalmente en el género masculino. Así, el consumo de estas sustancias está más extendido en los varones y se incrementa con la edad; (vi) el consumo de psicofármacos y tabaco es mayor en mujeres, mientras que el consumo de bebidas alcohólicas y de cannabis es mayor en los hombres jóvenes; (vii) el consumo de anfetaminas está más extendido en los hombres que en las mujeres y su consumo se incrementa con la edad; (viii) el riesgo percibido por las mujeres frente al consumo de sustancias psicoactivas es superior al percibido por los hombres tanto en el consumo esporádico como habitual (Martínez, 2006; Navarro, 2007; Fernández, 2010; Laespada, 2000; Vielva, 2000; Tirado, Aguaded y Marín, 2009; Laespada et al., 2004; CICAD/OEA, 2013; Romo, 2004).

Con base en lo anterior nos propusimos identificar entre la población universitaria analizada, losaspectos delas prácticas deconsumo de sustancias psicoactivas que se asocian al género como elemento diferenciador.

\section{Apuesta metodológica}

Para acercarnos a los objetivos planteados se propuso un estudio descriptivo-interpretativo, considerando un diseño mixto (Creswell and Plano, 2011) a partir del cual nos acercamos al objeto de estudio. Siguiendo a Tashakkori y Creswell (2007), los diseños mixtos son un tipo de investigación "en la cual se reúnen y analizan datos, se integran los 
hallazgos y se proponen inferencias usando aproximaciones cualitativas y cuantitativas" (p. 4). Este tipo de diseño recoge y analiza rigurosamente datos cualitativos y cuantitativos basados en preguntas de investigación o preguntas teóricas. Combina las distintas formas de datos de manera secuencial, dando prioridad a lo cualitativo o a lo cuantitativo y permite ampliar la investigación con un segundo método, lo que se convierte en una de sus ventajas en tanto con frecuencia percibimos que una sola fuente no es suficiente para dar cuenta del problema. Entre las tipologías propuestas en la bibliografía para los diseños mixtos, este trabajo consideró un modelo secuencial explicativo (Creswell and Plano, 2011).

Este modelo propone desarrollar la investigación en dos etapas: una etapa cuantitativa, seguida de una etapa cualitativa que permita la interpretación de los datos. En otras palabras, el propósito de este diseño es que los datos cualitativos ayuden a explicar o construir los resultados de la fase cuantitativa (Creswell and Plano, 2011). Una de las características es que las fases se conducen de manera separada y se recolecta solamente un tipo de datos a la vez. El reto hace referencia a que el diseño requiere tiempo para lograr integrar las dos fases, ya que la fase cualitativa puede tomar más tiempo que la fase cuantitativa. A continuación, se describe cómo se llevó a cabo cada una de las fases mencionadas anteriormente.

\section{Fase de investigación cuantitativa}

La etapa se desarrolló a partir del análisis descriptivo e inferencial de la información de una universidad colombiana participante en el II Estudio epidemiológico sobre uso de drogas en población universitaria, realizado entre 2012 y 2013 en los países miembros de la Comunidad Andina². Según el diseño metodológico propuesto, este momento de análisis cuantitativo fue considerado como una etapa previa descriptiva; lo que nos permitió pensar el contexto universitario y sus prácticas de modo general, observando las tendencias con mayor certeza. A su vez, no solo generó una descripción de los procesos de consumo en universitarios sino un marco problematizador que le dio sentido a la segunda fase de esta investigación; fase que pretendió indagar por las prácticas de consumo de sustancias psicoactivas entre universitarios e identificar, entre otros objetivos, las diferencias en el comportamiento frente al consumo ${ }^{3}$ asociadas al género.

\footnotetext{
${ }^{2}$ La base de datos del II Estudio epidemiológico en población universitaria 2012, para la universidad objeto de estudio, fue suministrada directamente por la OEA con el formulario de aplicación correspondiente.

${ }^{3}$ Para ampliar la información sobre la fase cuantitativa de la investigación consultar Espinosa-Herrera, Castellanos-Obregón y Osorio-García (2016).
} 


\section{Fase de investigación cualitativa}

El enfoque cualitativo permitió dejar hablar, escuchar y comprender las prácticas de consumo como fruto de un proceso de construcción a partir de las múltiples lógicas presentes en los diversos y heterogéneos actores sociales. Se recurrió a la perspectiva etnográfica como parte de los métodos comprensivos de investigación social. Esta decisión se apoyó en lo propuesto por Galeano y Vélez (2000), quienes plantean que los estudios etnográficos permiten captar el punto de vista de un grupo social concreto describiendo las acciones y los hechos que se desarrollan en dicho contexto; propiciando la reflexión de las personas sobre las creencias, prácticas y sentimientos e identificando el sentido actual que tienen las mismas.

Para realizar la construcción de unos datos confiables y un conocimiento pertinente sobre el objeto de estudio focalizado, se utilizaron diversas herramientas: observación participativa, entrevista semiestructurada y grupos de enfoque. Con el ejercicio de observación se identificó un grupo de estudiantes consumidores activos ${ }^{4}$, los territorios donde los jóvenes consumen, las actividades que se articulan a ese consumo, los temas de conversación entre ellos y la particularidad de los lenguajes entre otros elementos.

Por medio de la observación en los espacios de consumo, invitamos a los estudiantes a participar en el estudio; para ello se les solicitó, a modo de muestreo de cadena o por redes ("bola de nieve") (Hernández Sampieri, Fernández Collado y Baptista Lucio, 2014), identificar a otras personas pertenecientes a la población de interés que quisieran participar en el trabajo. Los estudiantes invitados pertenecen a diferentes programas académicos de la universidad regional objeto de estudio. Por su parte, los criterios de selección fueron: (i) estudiantes matriculados en cualquier pregrado de dicha universidad; (ii) estudiantes consumidores activos de sustancias psicoactivas.

La riqueza de la información llevó a la necesidad de profundizar con entrevistas semiestructuradas individuales para comparar las experiencias de consumo, en especial sobre los aspectos relacionados con el consumo de psicoactivas y el género de los universitarios.

El análisis del trabajo se realizó desde una lógica abductiva; es decir que al partir de la teoría se hizo el análisis de los datos, pero con un retorno para unir los dos análisis metodológicos. Este ejercicio demandó estrategias en la manera como se ordenaron los argumentos y explicaciones en tanto cada vez que se construyó un concepto, se realizó un proceso de inducción

\footnotetext{
${ }^{4}$ El grupo de consumidores activos estaba constituido por 12 estudiantes.
} 
analítica que debió demostrarse. A continuación, se presentan los resultados de la indagación.

\section{Resultados}

Al comparar los hallazgos de este trabajo con estudios anteriores se encuentra que algunos de los elementos propuestos, respecto a los consumos de sustancia psicoactivas diferenciados por género, quedan en evidencia.

Los resultados coinciden con el planteamiento de que en la población joven aparece un incremento del consumo entre las mujeres especialmente de alcohol, marihuana y drogas de prescripción médica; sin embargo, se evidenció que las cifras de consumo en los hombres continúan siendo superiores. En este sentido frente a los datos entregados por el I Estudio epidemiológico sobre uso de drogas en población universitaria (2009), y teniendo en cuenta de que el análisis para este trabajo se realizó con la base de datos del II Estudio epidemiológico sobre uso de drogas en población universitaria (2012), el estudio evidenció un incremento del 61 \% en el uso del alcohol principalmente en mujeres. El uso de marihuana mostró una variación estadísticamente significativa desde el $11 \%$ en el año 2009 a $15 \%$ en 2012, situación más evidente en hombres. En sustancias como el alcohol y el tabaco el consumo aumentó en general, pasando entre los estudiantes universitarios hombres de $72,5 \%$ a $78,3 \%$ y entre las mujeres de $57,4 \%$ a $65,9 \%$. El consumo de marihuana en hombres creció de 6,6 \% a 10,9\% y entre las mujeres de 3,3\% a 5,4\% (CICAD/OEA, 2013).

Entre los consumidores de drogas ilegales predomina el sexo masculino, mientras que encontramos que las mujeres inician el consumo de algunas sustancias de manera más tardía que los hombres. Lo anterior se aprecia en la figura 1 en la cual se muestra que entre las mujeres la edad de inicio de consumo de casi todas las sustancias psicoactivas ${ }^{5}$ consideradas para este estudio (alcohol, cigarrillo, marihuana, cocaína, éxtasis, LSD y hongos) está por encima de la edad de inicio de los hombres.

\footnotetext{
${ }^{5}$ Utilizando el percentil 50 de las edades del primer consumo.
} 


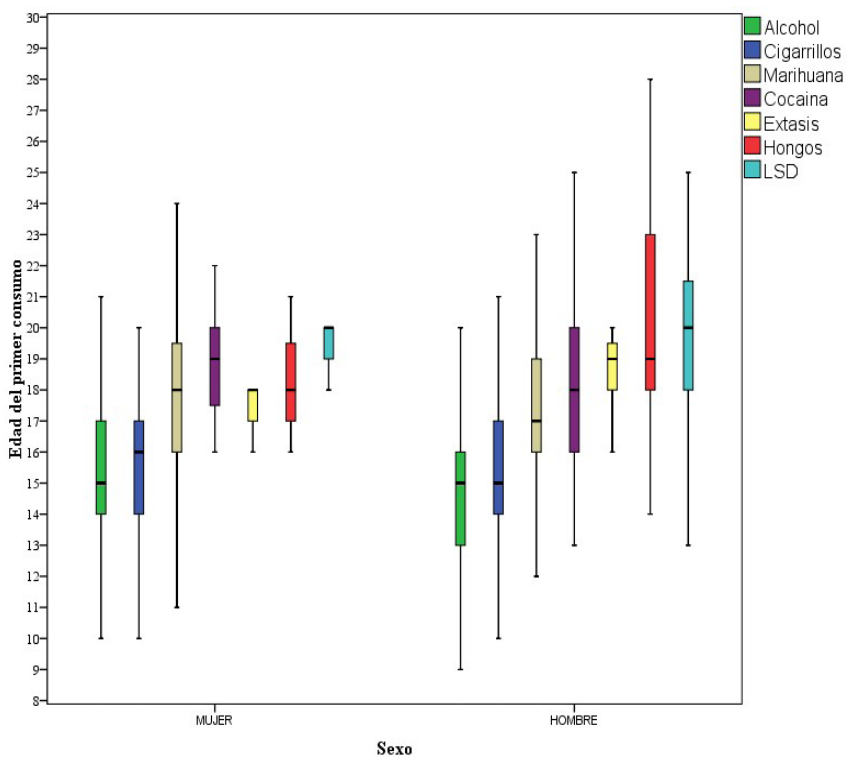

Figura 1. Inicio y escalamiento en el consumo de las sustancias psicoactivas, diferenciado por género. Fuente: análisis propio de la autora a partir de la base de datos del II Estudio epidemiológico andino sobre consumo de drogas en población universitaria (región Colombia).

El uso de los hongos y el LSD en mujeres es mucho menor (9 mujeres/65 hombres), ellas inician más tarde en el consumo de estas sustancias y la tendencia apunta a abandonar su uso más temprano que los hombres.

Los hombres inician más temprano en el uso de cigarrillo, marihuana y cocaína; mientras que con el alcohol, hombres y mujeres inician a la misma edad. En este sentido puede plantearse que no existen diferencias significativas en la edad de inicio del uso de cigarrillo, aunque los hombres son en promedio un año más precoces. Lo anterior corresponde con Romo (2004) cuando plantea que la tendencia a la equiparación de los hábitos de consumo entre hombres y mujeres en algunas sustancias es cada vez más perceptible.

Ahora bien, las diferencias en los consumos de sustancias psicoactivas entre ambos géneros no es solamente numérica. Como se plantea en el apartado de aproximación teórica, existen elementos culturales que impactan en el consumo de sustancias psicoactivas de hombres y mujeres. La percepción de las mujeres es que consumir sustancias psicoactivas no es valorado del mismo modo por la sociedad, aún entre población joven. Mientras que el consumo en hombres es percibido como una conducta 
natural, social y culturalmente aceptada, para las mujeres supone una práctica trasgresora de los valores sociales dominantes. Por ello, "las mujeres consumidoras sobrellevan un mayor grado de sanción respecto a los hombres, lo cual se materializa en tensiones y conflictos familiares, el rechazo por parte del entorno social más próximo y un menor apoyo familiar o social" (entrevista 5).

Esta valoración social desigual para el consumo en cada uno de los géneros, establece que hacia las mujeres exista una mayor estigmatización y señalamiento social.

Consumir drogas puede representar una forma de reto a los valores sociales dominantes, lo que trae consigo un mayor grado de reproche social, tenemos que soportar que nos señalen mientras que a los hombres le celebran la hombría y la masculinidad por consumir. (Entrevista 8)

Estas diferencias están relacionadas con los imaginarios construidos a través de la historia que determinan una carga simbólica mucho más fuerte hacia la mujer, "el hecho de ver a una mujer fumando, rascando, pegando o vendiendo marihuana, implica una mayor censura social" (entrevista 5), señalándola como 'marihuanera' y comparándola con vagos y desocupados.

Entre ambos géneros se constata la importancia de la experimentación previa con la intención de consumo futuro. Sin embargo, mientras las mujeres son inducidas al consumo por sus parejas o amigos, los hombres asocian el consumo a los imaginarios de masculinidad y hombría. Lo anterior implica asumir riesgos, por el miedo al ridículo ante las presiones del grupo. Asimismo, el inicio tardío de las mujeres en el consumo se relaciona también con el hecho de que ellas reciben menor libertad y mayor control por parte del grupo familiar.

Los testimonios evidencian que la entrada a la universidad y los primeros años de vida universitaria se corresponden con un incremento en el consumo y en la diversificación o tránsito hacia otras sustancias. La diversificación o policonsumo es un rasgo característico, en especial entre los consumidores masculinos "cuando una mujer muestra hábitos de consumir mucho o varias sustancias al tiempo, los mismos compañeros comienzan a señalarte como que eres muy muela o un colino"6 (entrevista 8).

La percepción de riesgo al consumir sustancias psicoactivas en hombres, es menor que la percepción en mujeres; sin embargo, en sustancias como marihuana, los consumidores de ambos géneros manifiestan una baja percepción de riesgo. Esto coincide con CICAD/OEA (2013) cuando

\footnotetext{
${ }^{6}$ Ser muela o colino hace referencia a una persona que consume marihuana en exceso.
} 
plantea que, en los países participantes del estudio (incluidos los del Cono Sur), Colombia presenta la mayor tasa de consumo de marihuana en la Región Andina; además, los estudiantes colombianos presentan la menor percepción de riesgo frente al consumo de esta sustancia amparados en argumentos como la no dependencia, no embriaguez o como medicina natural que no tiene químicos y en sus usos terapéuticos.

\section{Conclusiones}

Este trabajo se propuso abordar los comportamientos diferenciados por género frente al consumo de sustancias psicoactivas. Para ello realizamos un proceso de reanálisis a partir de los datos desagregados de una universidad regional colombiana, seleccionada para participar en el II Estudio epidemiológico sobre uso de drogas en población universitaria para el año 2012 y entrevistas a consumidores de sustancias psicoactivas de la misma institución.

Los hallazgos muestran coincidencias con estudios anteriores, evidenciando que el consumo de sustancias psicoactivas tiene una relación directa con la condición de género como un elemento diferenciador de las prácticas. En este sentido existen dos lecturas frente al consumo de este tipo de sustancias: las relacionadas con población adulta, específicamente en mujeres, y las relacionadas con población joven donde el consumo se percibe como expresión de la igualdad de género. Sin embargo esta última lectura no muestra una igualdad total frente al tema, ya que las mujeres son en ocasiones señaladas y estigmatizadas.

También se encuentra que las diferencias por género se reflejan en la edad de inicio del consumo, las diferentes sustancias que se consumen, los niveles de consumo, las motivaciones para el inicio, las percepciones sociales y las construcciones de género.

Las prácticas de consumo siguen siendo masculinas; sin embargo, en el contexto universitario, es evidente que se acortan las distancias y tendencias entre hombres y mujeres.

Se concluye que, si bien existen cambios en el consumo de sustancias psicoactivas por género entre los jóvenes y cambios en la percepción que se tiene frente a este tipo de consumo, el rechazo social hacia las mujeres consumidoras sigue siendo un elemento diferenciador de este tipo de prácticas. En este sentido, a modo de reflexión, se reconoce la necesidad de plantear otras investigaciones que indaguen sobre los factores asociados al inicio del consumo de sustancias psicoactivas en mujeres y a la recurrencia en este tipo de prácticas especialmente con algunas sustancias. Igualmente 
es necesario indagar por los factores que se asocian con los procesos de adicción en mujeres, así como los efectos o consecuencias que el consumo tiene con otras aristas de lo cotidiano, más allá de su relación con la salud.

\section{Referencias bibliográficas}

Castellanos, J.M. y Espinosa, G. (2013). Revisión de las tendencias de investigación sobre consumo de sustancias ilegales por los jóvenes. Revista de Antropología y Sociología: VIRAJES, 15 (2), 15-55.

CICAD/OEA. (2013). II Estudio Epidemiológico Andino sobre consumo de drogas en población universitaria. Informe regional. Recuperado de http://www.comunidadandina.org/ StaticFiles/20132718338Informe Regional.pdf.

Creswell, J. and Plano, V. (2011). Designing and Conducting Mixed Methods Research. Thousand Oaks, USA: SAGE Publications.

Espinosa-Herrera, G., Castellanos-Obregón, J.M. y Osorio-García, D. (2016). Condición juvenil y drogas en universitarios: el caso de una universidad regional. Revista Latinoamericana de Ciencias Sociales, Niñez y Juventud, 14 (2), 1451-1468.

Espinosa, G., Montoya, L.D. y Beltrán, G.L. (2015). Aproximación al uso de la marihuana en el ámbito universitario: caso Universidad de Caldas. Revista de Antropología y Sociología: VIRAJES, 17 (2), 235-258.

Fernández Bustos, P. (2010). Estudio de los factores de riesgo y protección del consumo (tesis de posgrado). Universidad de Alcalá, Alcalá, España.

Galeano, M. y Vélez, O. (2000). Investigación cualitativa. Estado del arte. Medellín, Colombia: Fondo Editorial Universidad de Antioquia.

Hernández Sampieri, R., Fernández Collado, C. y Baptista Lucio, M. (2014). Metodología de la investigación. Ciudad de México, México: McGraw-Hill.

Laespada, T. (2000). Alcohol y tabaco. Bilbao, España: Servicio Central de Publicaciones del Gobierno Vasco.

Laespada, T., Iraurgi, J. y Arostege, E. (2004). Factores de riesgo de protección frente al consumo de drogas: hacia un modelo explicativo del consumo de drogas en jóvenes de la CAPV. Bilbao, España: Universidad de Deusto.

Martínez, J.M. (2006). Factores de riesgo y protección ante el consumo de drogas y representaciones sociales sobre el uso de éstas en adolescentes y adultos jóvenes (tesis de posgrado). Universidad de Granada, Granada, España.

Navarro, B. (2007). Consumo de drogas en jóvenes y conducción de vehículos (tesis de posgrado). Universidad de Granada, Granada, España.

Pandina, R.J. (2002). Risk and Protective Factor Models in Adolescent Drug Use: Putting Them to Work for Prevention. Recuperado de https://archives.drugabuse.gov/meetings/CODA/ Risk.html.

Parra, R. (1996). La universidad. Escuela y modernidad en Colombia. Bogotá, Colombia: Tercer Mundo Editores.

Romani, O. (1999). Las drogas. Sueños y razones. Barcelona, España: Ariel.

Romo, N. (2004). Género y uso de drogas: la invisibilidad de las mujeres. Humanitas: Humanidades Médicas, 5, 69-83.

Sánchez, L. (2008). Drogas e perspectiva de xénero. Documento marco. Plan de atención integral á saúde da muller de Galicia, España.

Tashakkori, A. and Creswell, J.W. (2007). The new era of mixed methods. Journal of Mixed Methods Research, 1 (3), 3-7. 
Tirado, R., Aguaded, J.I. y Marín, I. (2009). Factores de protección y de riesgo del consumo de alcohol en alumnos de la Universidad de Huelva. Huelva, España: Universidad de Huelva.

Vielva, I. (2000). Drogas ilegales. Bilbao, España: Servicio Central de Publicaciones del Gobierno Vasco. 